\title{
Zwischen Pandemie und unternehmerischem Aufbruch
}

\author{
Hans Kurt ${ }^{a}$, Sandra Ziegler ${ }^{b}$ \\ ${ }^{a}$ Dr. med., Präsident des Verwaltungsrats $\mathrm{EMH}^{\mathrm{b}}{ }^{\mathrm{b}}$ Dr. phil. II, Geschäftsführerin $\mathrm{EMH}$
}

Die weltweite COVID-19-Pandemie und die verhängten Massnahmen zu deren Eindämmung gingen nicht spurlos an der EMH Schweizerischer Ärzteverlag AG vorbei. Der Printwerbemarkt und der Stellenmarkt brachen wegen der wirtschaftlichen Folgen der Pandemie ein. Dank den im März umgehend eingeführten Sparmassnahmen konnte aber diesen COVID-Effekten erfolgreich begegnet werden. So ist es EMH im Berichtsjahr 2020 gelungen, ein positives Resultat vor Steuern (EBIT) von CHF $677293 \mathrm{zu}$ erzielen, das sogar leicht über dem Niveau des Vorjahres liegt. Dies, obwohl der Umsatz gegenüber dem Vorjahr um 17,3\% tiefer war. Aber nicht nur die finanziellen Folgen der Pandemie beschäftigten das Unternehmen, sondern auch die Konsequenzen für das Personal. Innert Kürze mussten sich Mitarbeiterinnen und Mitarbeiter, die sonst im Team zu arbeiten gewohnt waren, auf Homeoffice umstellen. Sitzungen fanden nur noch virtuell statt, und dies in einer Phase der Veränderung im Unternehmen.

\section{Konkurs des langjährigen Produktionspartners}

Geprägt wurde 2020 aber auch durch den Konkurs der Medienmacher AG im Januar. Dadurch verlor EMH ihren langjährigen Produktionspartner sowohl im Druck- als auch im digitalen Bereich. Mit der VogtSchild Druck AG und der snowflake productions gmbh konnte EMH noch im Januar zwei versierte Partner gewinnen, die halfen, die termingerechte und qualitativ hochstehende Herausgabe der Zeitschriften sicherzustellen. Einige Mitarbeiter und Mitarbeiterinnen der Medienmacher AG konnten in die EMH übernommen werden. Trotz dieser Herausforderungen blickt
EMH zuversichtlich in die Zukunft. Dies hat drei Gründe. Zum einen hat EMH in einem vertieften Strategieprozess die Eckpfeiler für die Entwicklung und den zu-

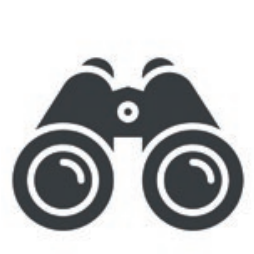

EMH hat in einem Strategieprozess die Eckpfeiler für die Entwicklung des Unternehmens erarbeitet. Fünf Handlungsfelder definieren über die nächsten Jahre die Prioritäten.

künftigen Erfolg des Unternehmens erarbeitet und das Vorgehen bestimmt, wie die neuen strategischen Ziele $\mathrm{zu}$ erreichen sind. Fünf Handlungsfelder in den Bereichen Communitys, Business Case, Angebotsentwicklung, Kommerzialisierung, Unternehmenskultur und Organisation definieren über die nächsten Jahre die Prioritäten im Unternehmen.

\section{Personelle Wechsel}

Mit der Verabschiedung der Strategie durch den Verwaltungsrat kam es auch zu einer Neuorganisation in der EMH-Geschäftsleitung. Dr. med. Natalie Marty,

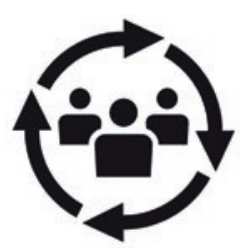

Im vergangenen Jahr kam es sowohl auf Ebene der Geschäftsleitung als auch im Verwaltungsrat zu personellen Wechseln.

langjährige Publizistische Leiterin und Geschäftsleitungsmitglied, wechselte in die Rolle eines Consultant medical publishing and open access. Wir sind froh, bleibt Natalie Marty EMH so weiter verbunden und können wir nach wie vor von ihrer grossen publizistischen Erfahrung profitieren. Sie war seit der Gründung von EMH mit dem Verlag verbunden. Wir danken ihr ganz herzlich für ihr langjähriges Engagement für den Schweizerischen Ärzteverlag.

Neu in der Geschäftsleitung vertreten sind Alexander Däppen, Leiter Marketing, und Martin Fux, Leiter Digital \& Business Development. Matthias Scholer, der als Chefredaktor der Schweizerischen Ärztezeitung bereits Mitglied der Geschäftsleitung war, übernimmt neu die Aufgaben des Leitenden Chefredaktors und ist in dieser Funktion für alle EMH-Zeitschriften zuständig. Daneben kam es auch zu einer Veränderung im EMHVerwaltungsrat. Anstelle des Ende 2019 zurückgetretenen Michael Düblins wurde neu Peter Riva als Vertreter der Petri Holding AG in den Verwaltungsrat gewählt.

\section{Digitalisierung verstärkt}

Zuversicht schöpfen wir aus der Tatsache, dass wir trotz der Krise auf zukunftsweisende Projekte gesetzt haben und diese erfolgreich umsetzen konnten. So haben wir während der Pandemie vorwiegend Digitalisierungsprojekte forciert. Das im letzten Jahr eingeführte neue Redaktionssystem Xpublisher verschlankt und vereinheitlicht die Publikationsprozesse im Verlag; neue, vorwiegend digitale Angebote erhöhen unsere Attraktivität bei den Werbekunden; und Aktivitäten im Bereich Sales Performance begleiten die Transition vom Verlagshaus zum Medienunternehmen.

\section{Starkes Team}

Und schliesslich sehen wir dank den Menschen im Unternehmen hoffnungsvoll in die Zukunft. Es erfüllt uns mit Stolz und Dankbarkeit, wie unsere Mitarbeitenden die grossen Herausforderungen durch die 
Pandemie mit viel Einsatz, Kreativität, Solidarität und Optimismus meistern. Sie sind es auch, die den herausfordernden Strategieprozess mitgetragen haben und nun umsetzen. Wir danken allen für ihren intensiven Einsatz. Dem Verwaltungsrat ist es aber auch ein Anliegen, der Geschäftsleitung, die sich 2020 durch ein starkes Krisenmanagement und eine weitsichtige Unternehmensführung ausgezeichnet hat, seinen Dank und seine Anerkennung auszusprechen.

\section{Herausforderungen der Zukunft}

Wir sind überzeugt, dass sich EMH mit der erarbeiteten Strategie und den eingeleite- ten Massnahmen zu einer noch stärkeren Unternehmung weiterentwickeln wird, die sich den Herausforderungen im Medienund Verlagsumfeld kreativ und erfolgreich stellen wird. Wir gehen jedoch auch davon aus, dass die Auswirkungen der COVID-19Pandemie noch länger spürbar sein werden. Dass wir in dieser herausfordernden Situation auch auf die aktive Unterstützung der beiden Aktionäre FMH Verbindung der Schweizer Ärztinnen und Ärzte und Petri Holding AG zählen können, schätzen wir ausserordentlich. Ein herzliches Dankeschön dafür.

kurt[at]solnet.ch
EMH - Das Wichtigste in Kürze

Der Schweizerische Ärzteverlag EMH wurde 1997 gegründet, um der medizinischen Publizistik in der Schweiz eine redaktionell unabhängige und fachlich relevante Plattform zu bieten. Die Aktiengesellschaft ist ein Gemeinschaftsunternehmen der Verbindung der Schweizer Ärztinnen und Ärzte FMH und der Petri Holding AG, dem mit Gründung 1488 ältesten Druck- und Verlagshaus der Welt. Die Kernprodukte Schweizerische Ärztezeitung und Swiss Medical Forum decken die zentralen Bereiche Standes- und Gesundheitspolitik sowie Aus-, Weiter- und Fortbildung ab. Um dieses Doppelgespann gruppiert sich eine Reihe weiterer Publikationen, welche unter anderem offizielle Organe wichtiger medizinischer Fachgesellschaften sind und deren fachliche und politische Kommunikationsbedürfnisse abdecken.

\section{Stellen schreibt man dort aus,} wo sie auch gefunden werden

Besuchen Sie die neue digitale Karriere-Plattform der SÄZ:

- Suchabonnemente mit Benachrichtigungen bei neuen Stellen

- Bewerbungsmöglichkeit und Bewerberverwaltung direkt im Portal

- Benutzerfreundlich auf allen Endgeräten

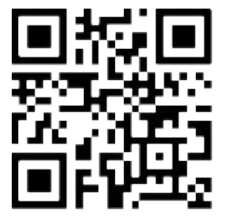

jobs.saez.ch

\section{Stellenmarkt}

Schweizerische Ärztezeitung

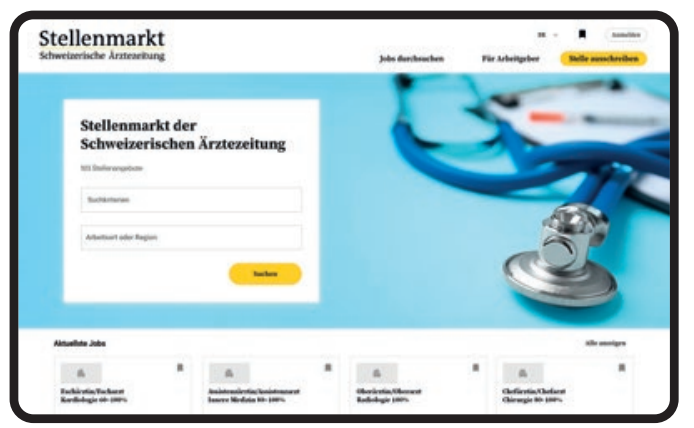

